\title{
NOUVELle
}

\section{Du cyclope à la réalité}

\section{Un nouveau regard sur la génétique de I'holoprosencéphalie}

Valérie Dupé ${ }^{1}$, Christèle Dubourg ${ }^{1,2}$, Marie de Tayrac ${ }^{1,2}$, Véronique David ${ }^{1,2}$
L'holoprosencéphalie, une pathologie hétérogène

La division anormale des deux hémisphères du cerveau pendant les premières semaines de la grossesse entraîne la survenue d'une maladie appelée holoprosencéphalie (HPE). C'est une maladie rare... toutefois pas si rare! En effet, cette pathologie est la malformation cérébrale congénitale la plus fréquente chez l'homme [1]. Elle est caractérisée par des altérations du clivage du cerveau antérieur et des anomalies faciales de la ligne médiane. Une holoprosencéphalie se développe lorsque la séparation des deux hémisphères cérébraux se réalise anormalement entre la $3^{e}$ et la $6^{\text {e }}$ semaine de la vie intra-utérine. L’holoprosencéphalie peut présenter différents degrés de sévérité : la forme alobaire $^{1}$, caractérisée par une absence complète de séparation des hémisphères cérébraux jusqu'à la présence d'un œil unique (communément appelée «cyclopie ») ; la forme semi-lobaire ; la forme lobaire, moins sévère; et enfin, des microformes, avec parfois une simple malformation labiale ou palatine, ou une incisive médiane unique, mais présentant toujours une déficience intellectuelle. La sévérité de la malformation cérébrale est corrélée à la précocité de l'apparition de l'anomalie au cours du développement.

L'holoprosencéphalie peut avoir pour origine des facteurs environnementaux

1 L'holoprosencéphalie alobaire, la plus sévère des formes classiques d'holoprosencéphalie est caractérisée par un ventricule cérébral unique avec absence de scissure interhémisphérique. comme le diabète insulino-dépendant et l'alcoolisme maternels. Elle peut également être la conséquence d'importantes anomalies chromosomiques (les trisomies 13 et 18), ou faire partie intégrante de syndromes polymalformatifs (comme ceux de Smith-Lemli-Opitz, PallisterHall et Hartsfield). Enfin, dans $40 \%$ des cas, elle peut être d'origine génétique. Cette pathologie présente une importante variabilité clinique qui résulte probablement de sa grande hétérogénéité génétique. Plusieurs gènes ont été impliqués dans l'HPE depuis 1996 et, à ce jour, des mutations ont été décrites dans 16 gènes différents: des gènes dits «majeurs » car impliqués dans plus de $1 \%$ des cas, et des gènes dits «mineurs » car mutés dans moins de $1 \%$ des cas [2]. Globalement, tout ces gènes appartiennent aux principales voies de signalisation orchestrant la formation du cerveau au cours du développement embryonnaire (comme SHH [sonic hedgehog], NODAL [membre de la famille du transforming growth factor $\beta$ ], FGF [fibroblastic growth factor] ou NOTCH [signalisation inter-cellulaire]) $[2,3]$.

Parmi ces gènes, l'un d'eux se distingue: sonic hedgehog. SHH est un gène qui code une molécule qui diffuse selon un gradient de concentration dans le tube neural pour permettre la différenciation des structures ventrales $[4,5](\rightarrow)$.

$(\rightarrow)$ Voir la Nouvelle de J. Ferent et al., $\mathrm{m} / \mathrm{s}$ $n^{\circ} 6-7$, juin-juillet 2014, page 705

La régulation de sa concentration est cruciale pour un développement cérébral correct et, lorsqu'elle
${ }^{1}$ UMR 6290 CNRS, institut de génétique et développement de Rennes, université de Rennesl, France.

${ }^{2}$ Service de génétique moléculaire et génomique, CHU Pontchaillou, 2, rue Henri Le Guilloux, 35033 Rennes, France. veronique.david@univ-rennesl.fr

n'est pas optimale, une holoprosencéphalie apparaît [6]. Tous les autres gènes impliqués dans cette pathologie interagissent fonctionnellement et ils sont, de près ou de loin, impliqués dans la régulation de la concentration de $\mathrm{SHH}$ (Figure 1A). L'hypothèse de notre équipe est qu'en régulant l'activité de SHH, les gènes impliqués dans l'holoprosencéphalie participent de façon concertée à l'apparition de la maladie. L'holoprosencéphalie serait donc une maladie du dosage de l'activité de SHH.

FGF, une nouvelle voie de signalisation majeure

Grâce aux centres labellisés « anomalies du développement », le CHU de Rennes a recruté la plus grande cohorte existant au niveau mondial, de patients atteints d'holoprosencéphalie. La mise à disposition de cette cohorte pour la recherche rend possible l'exploration des bases moléculaires de l'holoprosencéphalie d'origine génétique.

Auparavant, seuls les gènes considérés comme majeurs (SHH, SIX3 [six homeobox-3], ZIC2 [zinc-finger protein-2] et TGIF [TGFB-induced factor homeobox]) étaient examinés, pour le diagnostic, de façon séquentielle par la technique de séquençage de Sanger. L'arrivée des techniques de séquençage à haut débit (NGS, next generation sequencing) nous a permis d'étudier systématiquement 20 gènes déjà impliqués, ou suspectés de l'être, dans I'appa- $\quad(\rightarrow)$ Voir la Nouvelle rition de l'holopro- de D. Stoppa-Lyonnet sencéphalie [7] et C. Houdayer, $\mathrm{m} / \mathrm{s}$ $(\rightarrow) . \quad$ page 123 


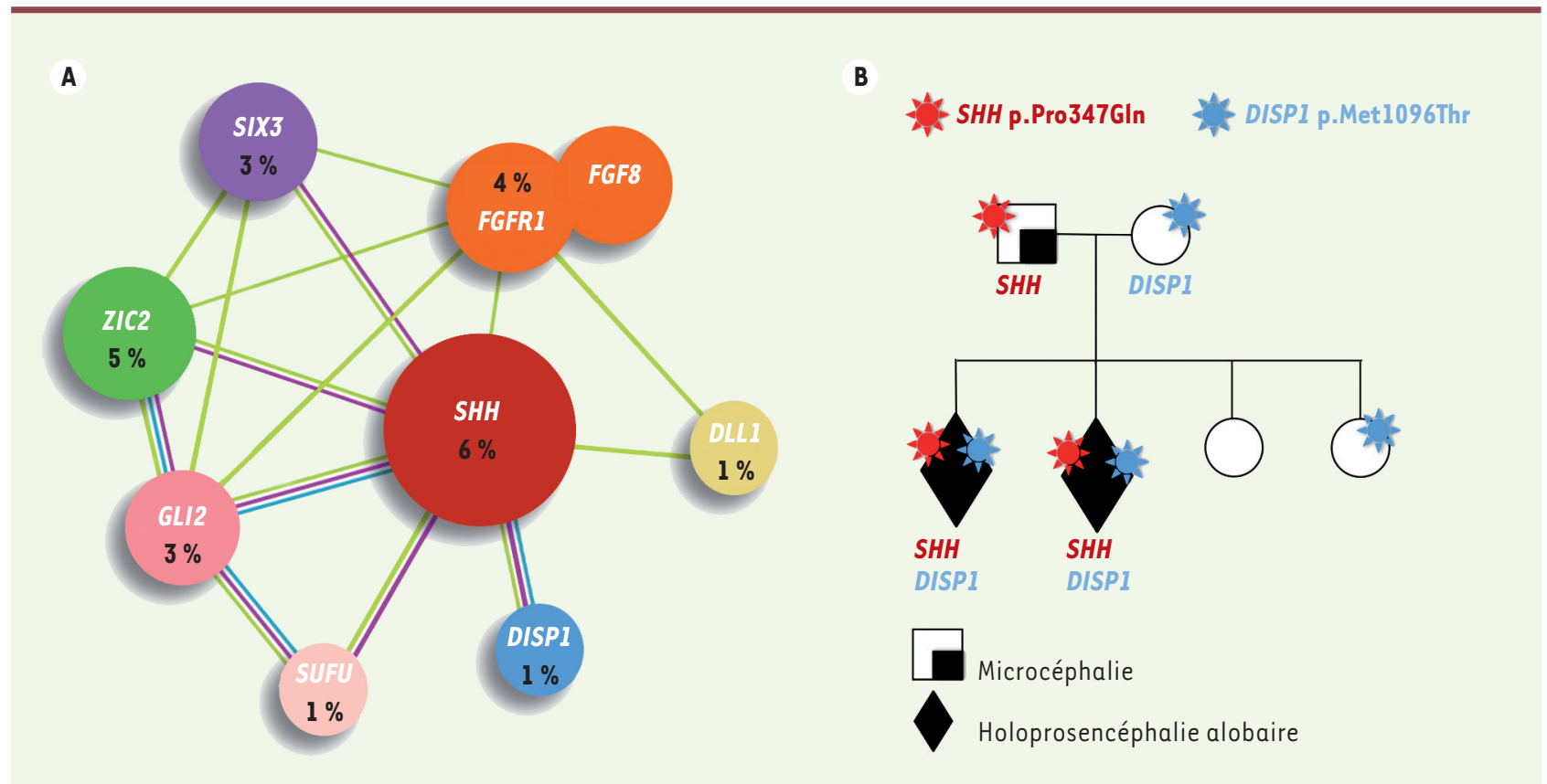

Figure 1. Génétique et transmission de l'holoprosencéphalie. A. Représentation simplifiée du réseau de gènes impliqués dans l'holoprosencéphalie. La fréquence des mutations délétères retrouvées chez 270 patients atteints d'holoprosencéphalie est indiquée pour chaque gène. DISPI: dispatched RND transporter family member 1 ; DLLI : delta-like canonical notch ligand 1 ; FGF : fibroblast growth factor ; FGFR : fibroblast growth factor receptor; GLI2 : zinc-finger protein; SHH : sonic hedgehog ; SIX3 : six homeobox-3; SUFU : negative regulator of hedgehog signaling ; ZIC2 : zinc-finger protein-2. B. Exemple de transmission digénique chez une famille atteinte d'holoprosencéphalie. SHH p.Pro347GIn: protéine sonic hedgehog mutée, la proline 347 est remplacée par une glutamine; DISPI p.Met1096Thr : protéine DISPI (dispatched RND transporter family member 1) mutée, le résidu méthionine 1096 est remplacé par une thréonine.

Cette étude, menée sur 270 patients, nous a permis d'affiner la fréquence des mutations pour chacun de ces gènes [8].

Ainsi, des mutations à effets délétères ont été identifiées dans environ $25 \%$ des cas et ont été retenues pour le diagnostic ; des variants moins significatifs ont également été observés dans $10 \%$ des cas, tous à l'état hétérozygote. Ces travaux, revisitant l'importance relative des gènes responsables de l'holoprosencéphalie, ont confirmé la complexité génétique de cette maladie.

Dans cette nouvelle classification, $\mathrm{SHH}$, ZIC2 et SIXZ restent les gènes majeurs en termes de fréquence. Ils sont suivis de près par GLI2 (zinc-finger protein), FGF8 (fibroblastic growth factor-8) et FGFRI (fibroblastic growth factor receptor-1), tandis que DLLI (delta-like canonical notch ligand 1), DISPI (dispatched RND transporter family member 1 ) et SUFU (negative regulator of hedgehog signa- ling) conservent leur statut de gènes mineurs (Figure IA).

Nous avons montré que les gènes codant les facteurs de croissance des fibroblastes (FGF) sont bien plus impliqués dans l'apparition de l'holoprosencéphalie que ce qui était attendu, très certainement parce qu'ils contrôlent la concentration de $\mathrm{SHH}$ disponible au niveau du cerveau. Ces résultats montrent l'importance de la voie de signalisation FGF et soulignent la nécessité de la considérer comme une voie majeure impliquée dans cette pathologie. Nous proposons que l'analyse des gènes codant le récepteur FGFRl et son ligand FGF8 soient systématiquement inclus dans la démarche diagnostique.

\section{Nouveaux modes de transmission pour} l'holoprosencéphalie

Les travaux que nous avons réalisés nous ont également permis de reconsi- dérer le mode de transmission de cette pathologie, initialement décrit comme dominant dans les études épidémiologiques [9]. Des études familiales ont montré que la plupart des mutations, identifiées chez les patients porteurs d'une forme sévère, étaient héritées d'un parent atteint d'une forme mineure voire asymptomatique (70\% des mutations $\mathrm{SHH}$ et SIX3, et $30 \%$ de celles de ZIC2 sont héritées). La pénétrance de la maladie s'avère ainsi incomplète y compris au sein d'une même famille, ce qui suggère qu'au moins un évènement génétique supplémentaire est nécessaire à l'apparition de l'holoprosencéphalie. En accord avec cette possibilité, dans de nombreux modèles de souris transgéniques, les animaux ne développent pas d'holoprosencéphalie sévère lorsqu'un seul gène est inactivé, l'inactivation de deux gènes étant nécessaire pour entraîner un phénotype de cyclopie [10]. Chez l'homme, des 
cas de digénisme ${ }^{2}$ ont également été rapportés [1]. Ces différentes observations convergent vers l'existence d'une transmission digénique voire multigénique de l'holoprosencéphalie.

De nouveaux arguments en faveur de ce modèle ont été apportés grâce aux techniques de NGS. Notre équipe a ainsi montré que $16 \%$ des mutations étaient en fait associées à une deuxième altération (par exemple, les associations: $S H H$ / DISPI, FGF8/FGFR1, FGF8/DLL1, DLL1/SHH, DISPI/DISPI ou DISPI/SUFU). Pour certaines familles, une parfaite co-ségrégation des deux mutations avec la forme sévère de la pathologie a pu être mise en évidence, tandis que la présence d'une seule de ces mutations n'était associée qu'à une forme mineure, comme une microcéphalie isolée (Figure 1B) [11]. Plusieurs modes de transmission existent donc pour l'holoprosencéphalie. Le mode autosomique dominant se vérifie pour les mutations qui surviennent de novo (70\% des mutations de ZIC2, $30 \%$ des mutations de SHH et de SIX3). Mais nous avons pu identifier, dans les familles consanguines, un mode de transmission autosomique récessif pour certaines mutations, essentiellement au niveau de gènes mineurs [12]. Dans la plupart des familles, cependant, il s'agirait très certainement d'une hérédité multigénique où les effets des mutations engendreraient collectivement une diminution de la concentration de SHH en-deçà d'une valeur critique, ou valeur seuil, à partir de laquelle l'holoprosencéphalie apparaîtrait.

Nos résultats présagent ainsi des répercussions importantes sur le conseil géné-

${ }^{2}$ Digénisme : mutation de deux gènes différents. tique de l'holoprosencéphalie, qui repose aujourd'hui principalement sur l'imagerie fœtale. Ils doivent donc inciter à une grande prudence pour le diagnostic prénatal. À l'exception des mutations de novo, le diagnostic moléculaire ne peut plus se contenter de l'identification d'une seule mutation causative, mais doit considérer la possibilité d'une transmission multigénique. La démonstration d'un tel mode de transmission ne peut cependant s'appuyer uniquement sur des études familiales et nécessitera la mise en œuvre de tests fonctionnels dédiés.

\section{Conclusion}

Pour l'holoprosencéphalie, et plus généralement pour toutes les pathologies multigéniques, le NGS présente un intérêt majeur. L'étude simultanée d'un grand nombre de gènes a permis l'identification de plusieurs mutations chez un même patient. Ces mutations ont des répercussions délétères et elles seraient collectivement nécessaires pour provoquer l'apparition de l'anomalie. C'est l'existence d'une cohorte importante, constituée depuis plus de 20 ans au CHU de Rennes, qui rend exploitable ces données de la génétique moderne. Cette cohorte nous a permis d'évaluer à nouveau l'implication des gènes cibles et de mettre en évidence une nouvelle voie de signalisation dans l'holoprosencéphalie. Malgré ces avancées notables, $70 \%$ des cas d'holoprosencéphalie d'origine génétique n'ont pas de diagnostic moléculaire. Il est donc important de poursuivre la recherche de nouveaux gènes candidats pour cette pathologie. $\diamond$ From cyclops to reality: a fresh look at the genetics of holoprosencephaly

\section{REMERCIEMENTS}

Nos travaux sont soutenus par l'Agence de la biomédecine, le projet innovation du CHU de Rennes, la Fondation maladies rares et l'Agence nationale de la recherche. Nous remercions les cliniciens, les fotopathologistes, le laboratoire de génétique moléculaire du CHU de Rennes et l'ensemble de l'équipe Génétique des pathologies liées au développement de l'Institut de génétique et développement de Rennes (IGDR). Ce travail est dédié aux malades et à leurs familles.

\section{LIENS D'INTÉRÊT}

Les auteurs déclarent n'avoir aucun lien d'intérêt concernant les données publiées dans cet article.

\section{RÉFÉRENCES}

1. Mercier S, Dubourg C, Garcelon N, et al. New findings for phenotype-genotype correlations in a large European series of holoprosencephaly cases. J Med Genet $2011 ; 48: 752-60$.

2. Roessler $\varepsilon$, Muenke M. The molecular genetics of holoprosencephaly. Am J Med Genet C Semin Med Genet 2010 ; 154C : 52-61.

3. Dupé V, Rochard L, Mercier S, et al. NOTCH, a new signaling pathway implicated in holoprosencephaly. Hum Mol Genet 2011 ; 20 : 1122-31.

4. Blaess S, Szabo N, Haddad-Tovolli R, et al. Sonic hedgehog signaling in the development of the mouse hypothalamus. Front Neuroanat $2014 ; 8: 156$.

5. Ferent J, Ruat M, Traiffort $\varepsilon$. Division symétrique ou division asymétrique : Sonic Hedgehog contrôle le destin des cellules souches neuronales adultes. Med Sci (Paris) $2014 ; 30: 705-8$.

6. Mercier S, David V, Ratié L, et al. NODAL and SHH dosedependent double inhibition promotes an HPE-like phenotype in chick embryos. Dis Modes Mech 2013 ; 6 : 537-43.

7. Stoppa-Lyonnet D, HoudayerC. Séquençage de nouvelle génération en génétique médicale; Dugénotype au phénotype, un défi majeur.Med Sci (Paris) 2012;28:123-4.

8. Dubourg C, Carré W, Hamdi-Rozé H, et al. Mutational spectrum in holoprosencephaly shows that FGF is a new major signaling pathway. Hum Mutat 2016 ; 37 : 1329-39.

9. Odent S, Le Marec B, Munnich A, et al. Segregation analysis in nonsyndromic holoprosencephaly. Am J Med Gene 1998 ; 77 : 139-43.

10. Schachter KA, Krauss RS. Murine models of holoprosencephaly. Curr Top Dev Biol 2008 ; 84 : 139-70.

11. Mouden C, Dubourg C, Carré W, et al. Complex mode of inheritance in holoprosencephaly revealed by whole exome sequencing. Clin Genet 2016 ; 89 : 659-68.

12. Mouden C, de Tayrac M, Dubourg C, et al. Homozygous STIL mutation causes holoprosencephaly and microcephaly in two siblings. PLoS One 2015; 10 e0117418.

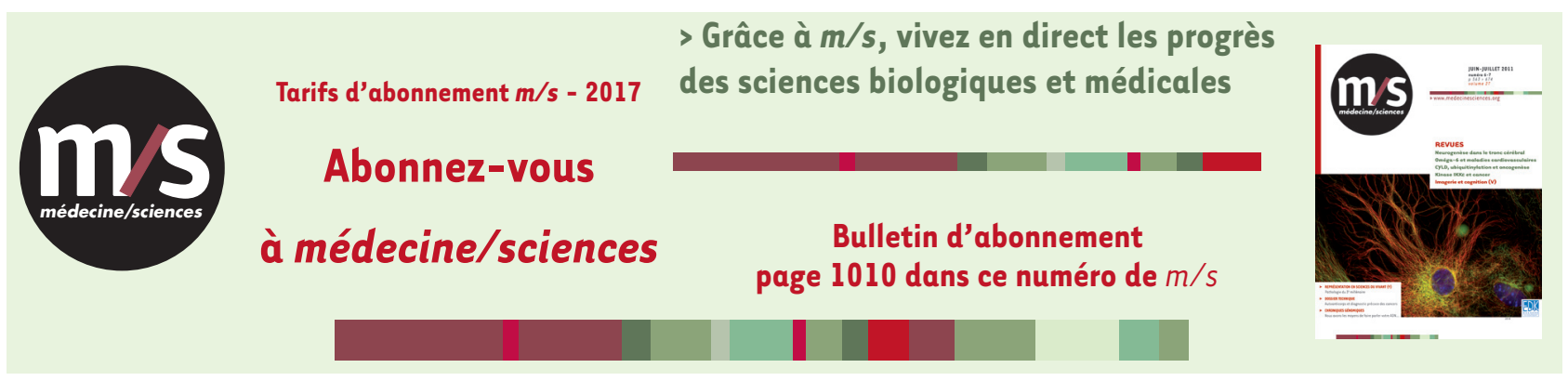

